\title{
Movie Mode Dynamic Transmission Electron Microscopy (DTEM): Multiple Frame Movies of Transient States in Materials with Nanosecond Time Resolution
}

\author{
Thomas LaGrange,* Bryan W. Reed,* William DeHope*, Richard Shuttlesworth* and Glenn \\ Huete*
}

*Lawrence Livermore National Laboratory, Physical and Life Science Directorate, Condensed Matter and Materials Division, 7000 East Avenue, P.O. 808 L-356

The ability to acquire high time resolution movies, dubbed Movie Mode Dynamic Transmission Electron Microscope (DTEM), expands the DTEM's science capabilities in single-shot mode by providing detailed histories of unique material events on the nanometer and nanosecond scale. Prior DTEM hardware only allowed single-pump/single-probe operation, building up a process's typical time history by repeating an experiment with varying time delays at different sample locations [1,2].

The Movie Mode DTEM upgrade enables single-pump/multi-probe operation [2]. It provides the ability to track the creation, motion, and interaction of individual defects, phase fronts, and chemical reaction fronts, providing invaluable information of the chemical, microstructural and atomic level features that influence the dynamics and kinetics of rapid material processes. For example, the potency of a nucleation site is governed by many factors related to defects, local chemistry, etc. While a single pump-probe snapshot provides statistical data about these factors, a multi-frame movie of a unique event allows all of the factors to be identified and the progress of nucleation and growth processes can be explored in detail. It provides unprecedented insight into the physics of rapid material processes from their early stages (e.g. nucleation) to completion, giving direct, unambiguous information regarding the dynamics of complex processes.

The two core components of the Movie Mode technology (Figure 1) are the arbitrary waveform generator (AWG) cathode laser system and a high-speed electrostatic deflector array. The AWG cathode drive laser enables continuously variable and controlled electron pulse durations from 250 $\mu$ s down to $5 \mathrm{~ns}$ in which a series of laser pulses is produced with user-defined pulse durations and delays that stimulates a defined photoemitted electron pulse train for a single sample drive event. Each pulse captures an image of the sample at a specific time. A fast-switching electrostatic deflector located below the sample directs each image to a separate patch on a large, high-resolution CCD camera. At the end of the experiment, the entire CCD image is read-out and segmented into a time-ordered series of images, i.e. a movie. The current technology produces 9 frame movies but near-term modification to the system should enable up to 25 -frame movies with interframe times as low as $25 \mathrm{~ns}$. This frame rate is six orders of magnitude faster than modern video-rate in situ TEM. Future versions of movie mode may also include fast-framing CCD technology, which can capture hundreds of frames within a few $\mu$ s. The operating principle of these devices is that the photoelectron CCD data from multiple frames is stored in on-chip buffers that are read out at the end of the acquisition. This presentation will discuss the Movie Mode DTEM technology in the context of materials science examples in which salient, irreversible events of rapid phase transformations are revealed through multiple frame movies.

References:

[1] T. LaGrange, M. R. Armstrong, K. Boyden, C. G. Brown, G. H. Campbell, J. D. Colvin, W. J. DeHope, A. M. Frank, D. J. Gibson, F. V. Hartemann, J. S. Kim, W. E. King, B. J. Pyke, B. W. 
Reed, M. D. Shirk, R. M. Shuttlesworth, B. C. Stuart, B. R. Torralva, and N. D. Browning, Applied Physics Letters 89, 044105 (2006).

[2] T. LaGrange, G.H. Campbell, B.W. Reed, M. Taheri, J.B. Pesavento, J.S. Kim, N.D. Browning, Ultramicroscopy 108, 1441 (2008)

[3] Work preformed at LLNL under the auspices of the U.S. Department of Energy by Lawrence Livermore National Laboratory under Contract DE-AC52-07NA27344 and supported in part by the U.S. Department of Energy, Office of Basic Energy Sciences, Division of Materials Sciences and Engineering.

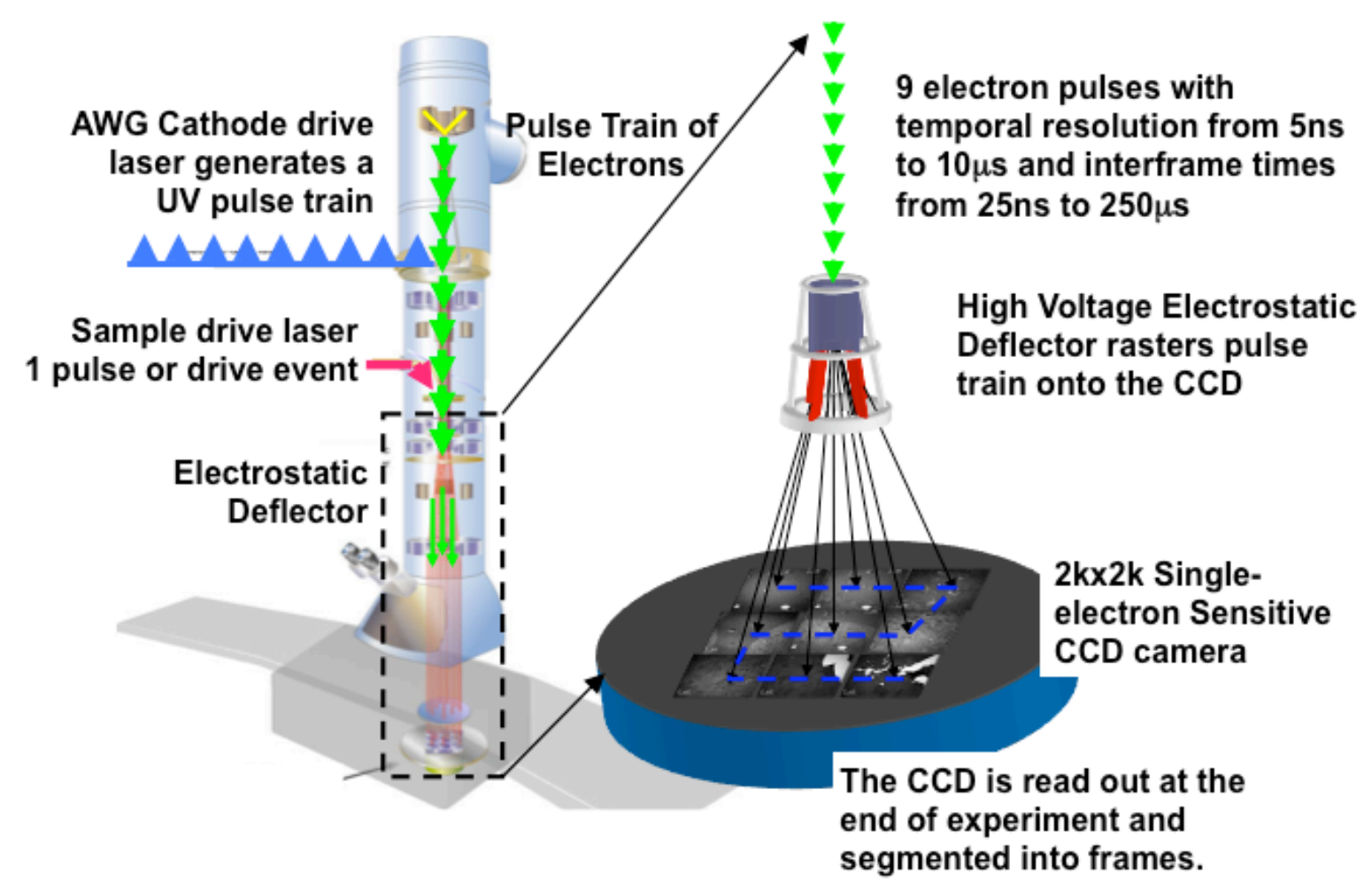

FIG 1. Schematic of the Movie Mode technology that enables single-pump/multi-probe operation and true, in-situ microscopy capabilities in the DTEM in which multi-frame movies of ultrafast material dynamics can be acquired. 